\title{
Tensions Ahead: How Do We Build Feasible Digital Health Systems That Are Person-Centred and Usable?
}

\author{
Helen ALMOND ${ }^{\mathrm{a}, 1}$, Karrie LONG ${ }^{\mathrm{b}}$ and Guillaume LEROUX ${ }^{\mathrm{c}}$ \\ ${ }^{a}$ Swinburne University of Technology and HISA NI-CoP \\ ${ }^{\mathrm{b}}$ Melbourne Health and Swinburne University of Technology and HISA UX-CoP \\ ${ }^{\mathrm{c}}$ University of South Australia and HISA NI-CoP
}

\begin{abstract}
Globally, the term person-centred care has become all-pervasive across healthcare delivery. It has been suggested that it has become synonymous with good quality care. The term is used frequently in the aims and objectives for healthcare service and provision, although what lies behind the rhetoric in terms of practice may be questionable. Further, in an environment which is becoming increasingly dependent on technology, the interaction needs the user experience of both consumer and healthcare provider. There is a challenge as national surveys of health professionals continue to criticize the usability of digital health interfaces. This panel discussion aims to highlight the factors, which should be considered when designing health information interfaces as person-centred for all users. Drawing on our panel's expertise, we suggest interfaces need to consider two main factors: personalization and user acceptance.
\end{abstract}

Keywords. Digital heath systems, person-centred, user experiences

\section{Introduction}

Recent national surveys of health professionals criticize the usability of digital health interfaces [1]. While there has been little improvement in this rating over time it has been identified that nurses and doctors have different needs with regards to the usability of systems as do consumers [2]. There remains much work to be done to design systems that meet the diverse needs of all users. User experience (UX) design should include awareness of the effects of interface and barriers to technology embedded in personcentred care (PCC), including both accessibility and usability. Designing for human users requires working with an amplified scope and sensitivity regarding heuristics and accessibility; thus accommodating the complexities of all potential users' physical limitations [3].

Furthermore, today's economy is fueled by highly competitive digital platforms and the uniqueness of each consumer. This potentializes user's satisfaction to even higher limits [4]. UX designer's tasks vary at the individual and organizational level but should demand designers be users' advocates and ensure users' needs are central to design and developmental efforts [3]. UX designers, using some form of PCC process, must focus evidence-based efforts to optimally address all efforts and user needs.

\footnotetext{
${ }^{1}$ Corresponding Author, Helen Almond; E-mail: halmond@swin.edu.au.
} 
UX designers, in healthcare, have to create products with which users have meaningful experiences. They should consider first Why? Assess each users' motivations for adopting a product, whether it relates to a task, and adds value. What? its essential functionality and finally How? accessibility aesthetics and not increasing workload [3].

Posing the results of the nation-wide usability-focused survey studies in Finland [1, 2] our international expert panel's experience will encourage and challenge our audience to join a discussion around the question: How do we build feasible digital health systems that are both person-centred and usable?

\section{Format}

The proposed format for the discussion: a) Facilitator gives brief introduction of panelists and discussion guidelines (10 minutes) b) Panelist position presentations (10 minutes each x $4=40$ minutes) c) Panel/Audience questions $\&$ answers (40 minutes).

The session will be audio recorded, the discussion to be thematically analyzed and disseminated as the outcomes of an international panel discussion on usability and person-centred care. At registration the audience will be informed of the recording, they will be reminded again when they enter the session, at which time they will consent for de-identified data collection (HREC 2019/7491 subject to amendment approval).

\subsection{Sample Questions (in addition to those from the audience)}

- How can digital health enhance person-centred care? How is can this be measured?

- User design, UX and PCC have similar goals but different outcomes. What are these? Are they simultaneously attainable and sustainable?

- Maintaining user design and PCC are imperatives for digital health systems. What culture is needed to nurture continuous usability improvement and integration? How can this culture be nurtured in private and public sectors?

- We are seeing novel roles and job descriptions in healthcare such as Clinical UX designer or Clinical Experience Manager. What skills and expertise are needed?

\section{The Panel}

\section{Facilitator Guillaume Leroux, Registered Nurse, Silver Chain Group}

Guillaume has nursed in Canada and Australia. He holds a Bachelor of Science in Nursing from the University of Montreal (Canada). During his career, he has been involved in diverse nursing leadership activities. Guillaume joined the NIA subcommittee in 2019, with a focus towards empowering the nursing workforce through digital health for ethically improved patient outcomes. 
Panelist 1 Timothy Kariotis, Research Fellow, Melbourne School of Government

Timothy holds a Master of Public Health from the University of Tasmania and is a current $\mathrm{PhD}$ candidate at the University of Melbourne School of Computing and Information Systems. He is fascinated with how technology can improve information sharing whilst meeting privacy expectations. Being a carer has shaped Timothy's focus; ensuring equity and justice in an increasingly digital information-driven society.

\section{Panelist 2 Dr Margaret Kennedy, Chief Nursing Information Officer, Gevity}

As a Managing Partner of Gevity, Margaret leads Gevity's Clinical Informatics Practice. Margie enables systems transformation supporting optimal health outcomes and quality clinical practice. Margie holds a PhD in Nursing, University of South Australia, a Master of Nursing, Bachelor of Science in Nursing and Post Graduate Certificate in Standards for Health Informatics from Canadian universities.

Panelist 3 Aimi Hino, Senior Product Designer, Medibank

From a nursing background Aimi co-founded Med Health Digital and now manages Designing for Health Australia, a community of practice for human-centred healthcare in Australia and NZ. Aimi holds a Bachelor of Science in Nursing, University of Queensland and General Assembly Certificate in User Experience. Her current interest is the future application of digital health, and the impact of focused communities.

Panelist 4 Ken Saman, CEO, Personify Care

Personify Care operates in Australia, NZ and US and is developing smart technology to prepare patients pre-surgery and monitoring recovery post-discharge enabling patients to get the best care beyond their hospital care. With his experience Ken's interest is in applying digital consumer trends to deliver on the potential of patient-centred care.

\section{References}

[1] Kaipio J, Lääveri T, Hyppönen H, Vainiomäki S, Reponen J, Kushniruk A et al. Usability problems do not heal by themselves: National survey on physicians' experiences with EHRs in Finland. International Journal of Medical Informatics. 2017;97:266-81.

[2] Kaipio J, Kuusisto A, Hyppönen H, Heponiemi T, Lääveri T. Physicians' and nurses' experiences on EHR usability: Comparison between the professional groups by employment sector and system brand. International Journal of Medical Informatics. 2019:104018.

[3] Friedman B, Hendry DG. Value sensitive design: Shaping technology with moral imagination. Mit Press; 2019.

[4] Kelly E. Work in the 21st century: Changing our mindsets. People and Strategy. 2008;31(4):20. 